\title{
Analysis of Apparent Diffusion Coefficients of the Brain in Healthy Controls: A Comparison Study between Single-Shot Echo-Planar Imaging and Read-out- Segmented Echo-Planar Imaging
}

\author{
Yangsean Choi, MD ${ }^{1}$, Eo-Jin Hwang, PhD ${ }^{1}$, Yoonho Nam, PhD ${ }^{1}$, Hyun Seok Choi, MD, PhD ${ }^{1,2}$, \\ Jinhee Jang, MD, PhD ${ }^{1}$, So-Lyung Jung, MD, PhD ${ }^{1}$, Kook-Jin Ahn, MD, PhD ${ }^{1}$, Bum-soo Kim, MD, PhD ${ }^{1}$ \\ ${ }^{1}$ Department of Radiology, Seoul St. Mary's Hospital, Seoul, Korea; ${ }^{2}$ Department of Radiology, Research Institute of Radiological Science, \\ Severance Hospital, Yonsei University College of Medicine, Seoul, Korea
}

Objective: To compare apparent diffusion coefficients (ADCs) of brain segments by using two diffusion-weighted imaging acquisition modes, single-shot echo-planar imaging (ss-EPI) and read-out-segmented echo-planar imaging (rs-EPI), and to assess their correlation and agreement in healthy controls.

Materials and Methods: T2-weighted (T2W) images, rs-EPI, and ss-EPI of 30 healthy subjects were acquired using a $3 \mathrm{~T}$ magnetic resonance scanner. The T2W images were co-registered to the rs-EPI and ss-EPI, which were then segmented into the gray matter (GM), white matter (WM), and cerebrospinal fluid (CSF) to generate masking templates. ADC maps of rs-EPI and ss-EPI were also segmented into the GM, WM, and CSF by using the generated templates. ADCs of rs-EPI and ss-EPI were compared using Student's $t$ tests and correlated using Pearson's correlation coefficients. Bland-Altman plots were used to assess the agreement between acquisitions.

Results: ADCs of rs-EPI and ss-EPI were significantly different in the GM $(p<0.001)$ and WM $(p<0.001)$. ADCs showed high agreement and correlation in the whole brain and CSF $(r>0.988 ; p<0.001)$. ADC of the WM showed the least correlation $(r$ $=0.894 ; p<0.001)$, and ADCs of the WM and GM showed poor agreement. Pearson's correlation equations for each brain segment were $y=1.1 x-59.4(G M), y=1.45 x-255(W M)$, and $y=0.98 x-63.5$ (CSF), where $x$ and $y$ indicated ADCs of rs-EPI and SS-EPI, respectively.

Conclusion: While ADCs of rs-EPI and ss-EPI showed high correlation and agreement in the whole brain and CSF, ADCs of the WM and GM showed significant differences and large variability, reflecting brain parenchymal inhomogeneity due to different regional microenvironments. ADCs of different acquisition methods should be interpreted carefully, especially in intraindividual comparisons.

Keywords: Apparent diffusion coefficient; Diffusion-weighted imaging; Single-shot echo-planar imaging; Read-out-segmented echo-planar imaging; Magnetic resonance imaging

\section{INTRODUCTION}

Diffusion-weighted imaging (DWI) is one of the most widely used sequences in daily radiological practice and image-based research. Its various clinical applications include predicting infarct cores in ischemic strokes (1-3), grading and differentiating abscesses and malignant brain tumors (4), and evaluating treatment responses in oncology

Received December 20, 2018; accepted after revision March 5, 2019.

Corresponding author: Hyun Seok Choi, MD, PhD, Department of Radiology, Seoul St. Mary's Hospital, 222 Banpo-daero, Seocho-gu, Seoul 06591, Korea; Department of Radiology, Research Institute of Radiological Science, Severance Hospital, Yonsei University College of Medicine, 50 Yonsei-ro, Seodaemun-gu, Seoul 03722, Korea.

- Tel: (822) 2228-7400•Fax: (822) 2227-8337• E-mail: hyunchoi@yonsei.ac.kr

This is an Open Access article distributed under the terms of the Creative Commons Attribution Non-Commercial License (https:// creativecommons.org/licenses/by-nc/4.0) which permits unrestricted non-commercial use, distribution, and reproduction in any medium, provided the original work is properly cited. 
(5-7). Apparent diffusion coefficient (ADC) is a quantitative metric derived from DWI, which provides numerical information on microscopic environments, such as cytotoxic edema (8), tumor cellularity (9), and white matter (WM) integrity (10). Because different tissues respond differently to the strengths and directions of diffusion gradients, DWI and ADC together play important roles in diagnosing diseases affecting various tissue types and having varying pathological processes.

Single-shot echo-planar imaging (ss-EPI) has been the most widely used DWI sequence in clinical practice (11). However, the advent of read-out-segmented echo-planar imaging (rs-EPI), which splits $\kappa$-space acquisitions into segments by using multiple shots, has reduced geometric distortion by a factor proportional to the segment width and T2*-induced blurring by accelerating $\kappa$-space traversals along the phase-encoding direction (12). Moreover, possible motion artifacts that might occur between each segment-induced phase error could be corrected by using navigator echoes (12). This method also promises improved diagnostic performances in numerous diseases, such as malignant hepatic tumors (13), sinonasal lesions (14), breast cancer (15), and prostate cancer (16). Although rs-EPI requires a longer acquisition time, its superior overall image quality and diagnostic performance support its use over ss-EPI (13-19).

Nevertheless, the conventional ss-EPI sequence cannot be ignored because of its short scan time, especially in patients with acute stroke. The benefits of each sequence should thus be scrutinized and applied appropriately, depending on the targeted diseases. Although several studies have emphasized the superior performance of rs-EPI over ss-EPI, most of them focused on a single disease and performed qualitative evaluations. Therefore, the purpose of our study was to quantitatively evaluate ADCs of the whole brain by using ss-EPI and rs-EPI and to determine the correlation and agreement between them in healthy controls.

\section{MATERIALS AND METHODS}

\section{Subjects}

The Institutional Review Board approved this retrospective study and waived the need for written informed consent. Between September 2012 and October 2013, 30 consecutive healthy adults with no medical history of neurological diseases (mean age $=64.4 \pm 14.2$ years; 15 men and 15 women) were included after reviewing their magnetic resonance (MR) images on the picture archiving and communication system at our institution. One radiologist with 10 years of experience in neuroradiology reviewed all the images and electronic medical records of the included subjects. Among the subjects, the indications for brain MR imaging were dizziness $(n=15)$, headache ( $n$ $=10)$, and wanted $(n=5)$.

\section{Image Acquisition}

MR images were acquired using a 3T MR scanner equipped with a 12-channel head coil (MAGNETOM Verio, Siemens Healthineers, Erlangen, Germany). Both ss-EPI and rs-EPI sequences were performed to acquire normal and highresolution axial diffusion-weighted images with gradient strength (b) values of 0 and $1000 \mathrm{~s} / \mathrm{mm}^{2}$, respectively. The acquisition parameters for both sequences were as follows: ss-EPI DWI sequence-repetition time (TR)/echo time $(\mathrm{TE})=6800 / 100 \mathrm{~ms}$, slice thickness $=5 \mathrm{~mm}$, slices $=26$, bandwidth $=1002 \mathrm{~Hz} / \mathrm{px}$, matrix $=192 \times 192$, field of view (FOV) $=220 \times 220 \mathrm{~mm}^{2}, \mathrm{~b}=0$ and $1000 \mathrm{~s} / \mathrm{mm}^{2}$, diffusion directions $=3$, read-out-segment $=1$, and mean acquisition time $=0.82 \mathrm{~min}$; $\mathrm{rs}$-EPI DWI sequence $-\mathrm{TR} / \mathrm{TE}=$ $4900 / 68 \mathrm{~ms}$, slice thickness $=5 \mathrm{~mm}$, slices $=26$, bandwidth $=651 \mathrm{~Hz} / \mathrm{px}$, matrix $=192 \times 192, \mathrm{FOV}=220 \times 220 \mathrm{~mm}$, $\mathrm{b}=0$ and $1000 \mathrm{~s} / \mathrm{mm}^{2}$, diffusion directions $=3$, read-outsegment $=9$, and mean acquisition time $=5.67 \mathrm{~min}$ (Table 1). Finally, axial $T 2$-weighted (T2W) images were acquired for image registration and segmentation with the following parameters: TR $=4900 \mathrm{~ms}, \mathrm{TE}=68 \mathrm{~ms}, \mathrm{FOV}=192 \times 192$ $\mathrm{mm}^{2}$, and slice thickness $=5 \mathrm{~mm}$.

\section{Image Processing and ADC Map Segmentation}

All image processing and automatic segmentation were performed using Statistical Parametric Mapping, version 8 (SPM8; Wellcome Department of Imaging Neuroscience, University College London, London, UK). The T2W images were co-registered to the rs-EPI at b-0 s/mm to be used as a reference. The rs-EPI at b-1000 s/mm $\mathrm{mm}^{2}$ and ss-EPI at $\mathrm{b}-0$ and $\mathrm{b}-1000 \mathrm{~s} / \mathrm{mm}^{2}$ were additionally co-registered to the reference image. The co-registered T2W images were then segmented into the gray matter (GM), WM, and cerebrospinal fluid (CSF) to generate masking templates, such that each voxel of the segmented GM, WM, and CSF represented the proportion of the three segments. By using the masking templates, ADC maps from the rs-EPI and ssEPI at $b-0$ and $b-1000 \mathrm{~s} / \mathrm{mm}^{2}$ were segmented into the GM, WM, and CSF, and into the combination of the GM and WM 
Table 1. Sequence Parameters for ss-EPI and rs-EPI

\begin{tabular}{lcc}
\hline \multicolumn{1}{c}{ Sequence Parameter } & rs-EPI & Ss-EPI \\
\hline Diffusion directions & Three-directions & Three-directions \\
b values $\left(\mathrm{s} / \mathrm{mm}^{2}\right.$ ) & 0,1000 & 0,1000 \\
Fat suppression & $0 \mathrm{n}$ & $0 \mathrm{n}$ \\
Repetition time (ms) & 4900 & 6800 \\
Echo time (ms) & 68 & 100 \\
Field of view (mm ${ }^{2}$ ) & $220 \times 220$ & $220 \times 220$ \\
Matrix & $192 \times 192$ & $192 \times 192$ \\
Slice thickness (mm) & 5 & 5 \\
Intersection gap (mm) & 1 & 1 \\
No. of slices & 26 & 26 \\
Phase-encoding direction & Anterior-posterior & Anterior-posterior \\
Echo spacing (msec) & 0.32 & 1.08 \\
Bandwidth (Hz/px) & 651 & 1002 \\
No. of read-out-segment & 9 & 1 \\
Mean acquisition time (min:sec) & $5: 40$ & $0: 49$ \\
\hline
\end{tabular}

rs-EPI = read-out-segmented echo-planar imaging , ss-EPI = single-shot echo-planar imaging

to represent the whole brain tissues.

\section{Statistical Analysis}

Student's $t$ test was used to compare ADCs between ss-EPI and rs-EPI within the same subjects. Pearson's correlation coefficients were calculated to determine the correlation between ADCs of ss-EPI and rs-EPI. Agreement of ADCs was assessed using Bland-Altman plots. All statistical analyses were performed using MATLAB (MathWorks, Natick, MA, USA) and $R$ version 3.2.2 (R Foundation, Vienna, Austria). The threshold for statistical significance was set at $p<0.05$.

\section{RESULTS}

Image Processing and ADC Mapping by Using ss-EPI and rs-EPI at b- 0 and b-1000 s/mm

The overall image quality of the acquired images was visually acceptable. The representative slices of rs-EPI and ss-EPI at b-0 and b-1000 s/mm ${ }^{2}$, corresponding ADC maps, and masks of brain regions acquired using the two different sequences are shown in Figure 1. The color-coded ADC map of rs-EPI showed less noise than did that of ss-EPI at b-1000 s/mm ${ }^{2}$, containing relatively homogeneous crosssectional $A D C$ values (blue) (Fig. 1).

Comparison of ADCs between ss-EPI and rs-EPI according to Brain Segments

Significant differences were found between the two ADCs in the GM $(p<0.001)$ and WM $(p<0.001)$, while no significant differences were found in the whole brain $(p=$
$0.091)$ and CSF $(p=0.700)$ (Table 2, Fig. 2). Furthermore, the difference between the two ADCs was greatest in the WM and smallest in the CSF (Fig. 2).

\section{Pearson's Correlation and Bland-Altman Plots for}

Agreement Assessment between ss-EPI and rs-EPI

The correlation coefficients of ADCs of rs-EPI and ss-EPI were all over 0.988 for the whole brain, GM, and CSF. The least correlation coefficient was seen in the WM $(r=0.894)$ (Fig. 3). Pearson's correlation equations for each brain segment were $y=1.1 x-59.4(G M), y=1.45 x-255$ (WM), and $y=0.98 x-63.5$ (CSF), where $x$ and $y$ indicated ADCs of rs-EPI and ss-EPI, respectively. The slope and intercept of the WM showed the largest variation apart from direct proportion. The mean difference and limits of agreement between the two ADCs were $54.5(19.4,89.6)$ for the whole brain, $26.9(-52.0,105.9)$ for the CSF, $67.4(31.5,103.3)$ for the WM, and $148.7(92.9,204.5)$ for the GM $\left(10^{-6} \mathrm{~mm}^{2} / \mathrm{s}\right)$, respectively (Fig. 4).

\section{DISCUSSION}

The advantages of rs-EPI are its improved spatial resolution and reduced susceptibility to artifacts, which compensate for the extended scan time. In this study, we quantitatively compared ADCs of rs-EPI and ss-EPI in the whole brain, GM, WM, and CSF of healthy controls to identify correlations and agreement between both sequences.

Overall, ADC values of ss-EPI were greater than those of rs-EPI throughout the brain segments, with significant 


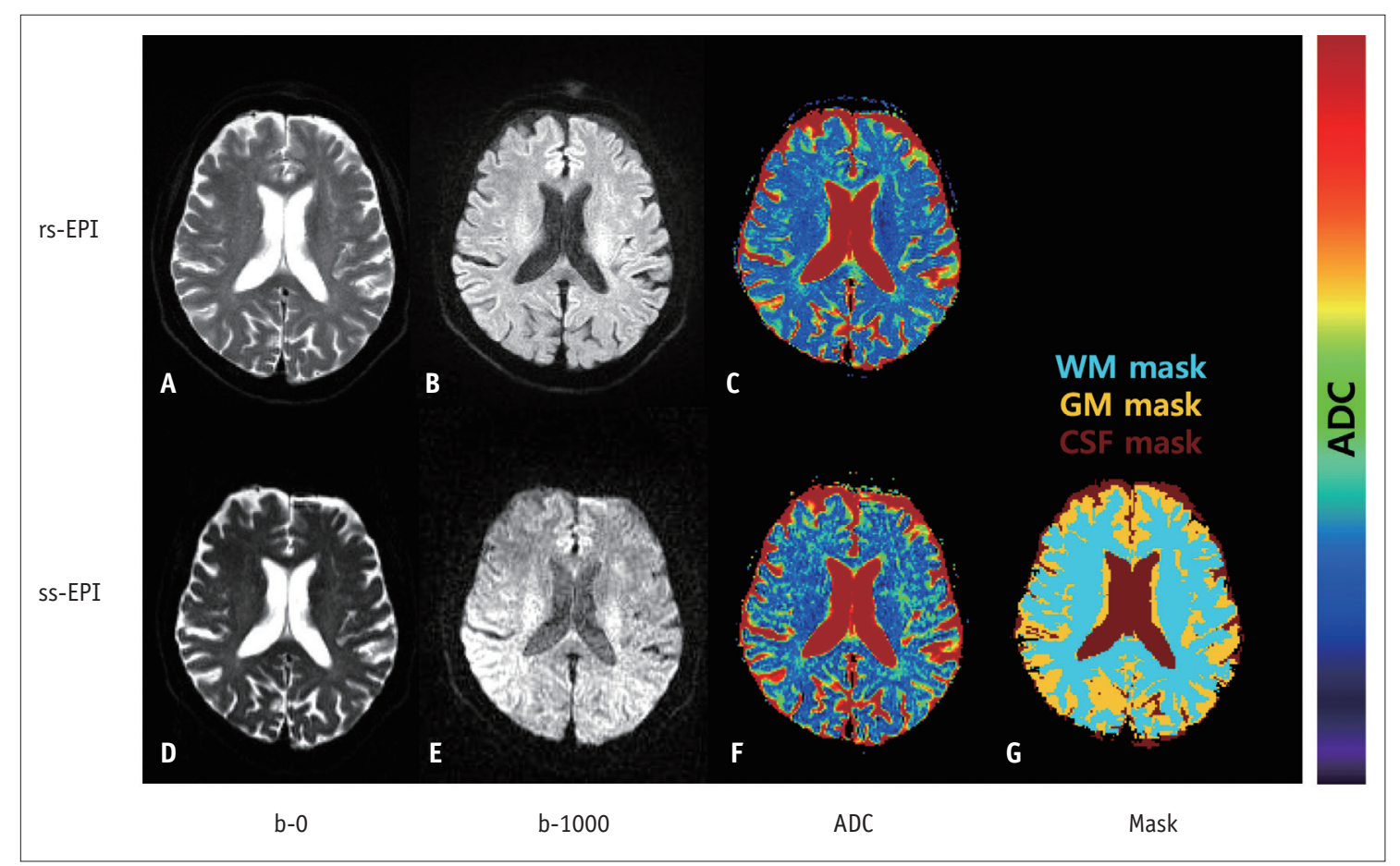

Fig. 1. Representative images of 55-year-old healthy subject.

A, B. rs-EPI at $b=0$ and $b=1000$. D, E. SS-EPI at $b=0$ and $b=1000$. ADC map of whole brain calculated using rs-EPI (C) and ss-EPI (F). G. Mask template of WM, GM, and CSF. ADC = apparent diffusion coefficient, CSF = cerebrospinal fluid, GM = gray matter, rs-EPI $=$ read-outsegmented echo-planar imaging, Ss-EPI = single-shot echo-planar imaging, $\mathrm{WM}=$ white matter

Table 2. Comparison of ADC from rs-EPI and ss-EPI

\begin{tabular}{lccc}
\hline \multicolumn{1}{c}{ Compartments } & rs-ADC & Ss-ADC & $P$ \\
\hline Whole brain & $1114.1 \pm 116.1$ & $1168.5 \pm 128.7$ & 0.09 \\
Gray matter & $977.3 \pm 122.9$ & $1126.0 \pm 142.8$ & $<0.001$ \\
White matter & $720.6 \pm 21.5$ & $788.0 \pm 34.8$ & $<0.001$ \\
Cerebrospinal fluid & $2249.0 \pm 266.4$ & $2275.9 \pm 265.1$ & 0.7 \\
\hline
\end{tabular}

All units are in $10^{-6} \mathrm{~mm}^{2} / \mathrm{sec}$. ADC = apparent diffusion coefficient

differences in the GM $(p<0.001)$ and WM $(p<0.001)$. Despite the differences, the two ADCs showed strong linear correlations in the whole brain, GM, and CSF $(r$ $>0.988)$, but relatively lower correlation in the WM ( $r$ $=0.894)$. Interestingly, ADCs of WM and GM showed variations-upward trends-between the two sequences on Bland-Altman plots. This finding may suggest that water diffusivity in the WM and GM was more influenced by the acquisition methods than was the diffusivity in the CSF, which essentially behaves like free water. One possible explanation is that water diffusion in biological tissues is non-Gaussian and distributes along the fine structures and geometric organization of neural tissues (20). More specifically, because the axon fibers in the brain parenchyma serve as barriers to translational motion (21), diffusion of water in neuronal tissues could be hindered by diffusion gradients, leading to a diffusion variation depending on the acquisition technique.

The microscopic heterogeneity of WM structures could also contribute to the largest $A D C$ variations between the two sequences. The WM is composed of axon bundles surrounded by complex extra-axonal components, such as astrocytes, glia, and randomly orientated extracellular matrix molecules (22). This complexity adds difficulty in characterizing the diffusion properties of the WM.

The acquisition of high $b$-value DWI also revealed that the simple mono-exponential behavior of the diffusion signal is no longer applicable in the WM (23). Instead, the diffusivity in the WM should be divided into a "fast" component, which arises from the hindered diffusion of the extra-axonal space, and a "slow" component, which is associated with restricted diffusion of the intra-axonal 


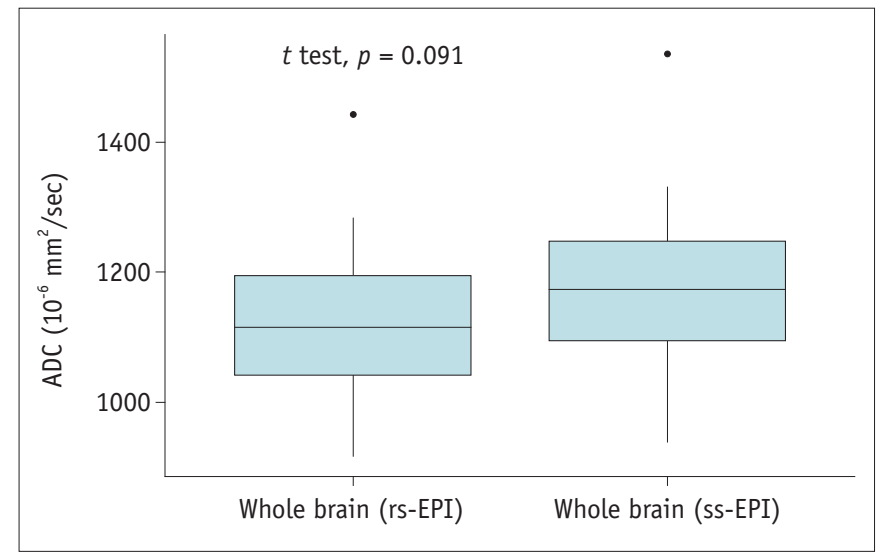

A
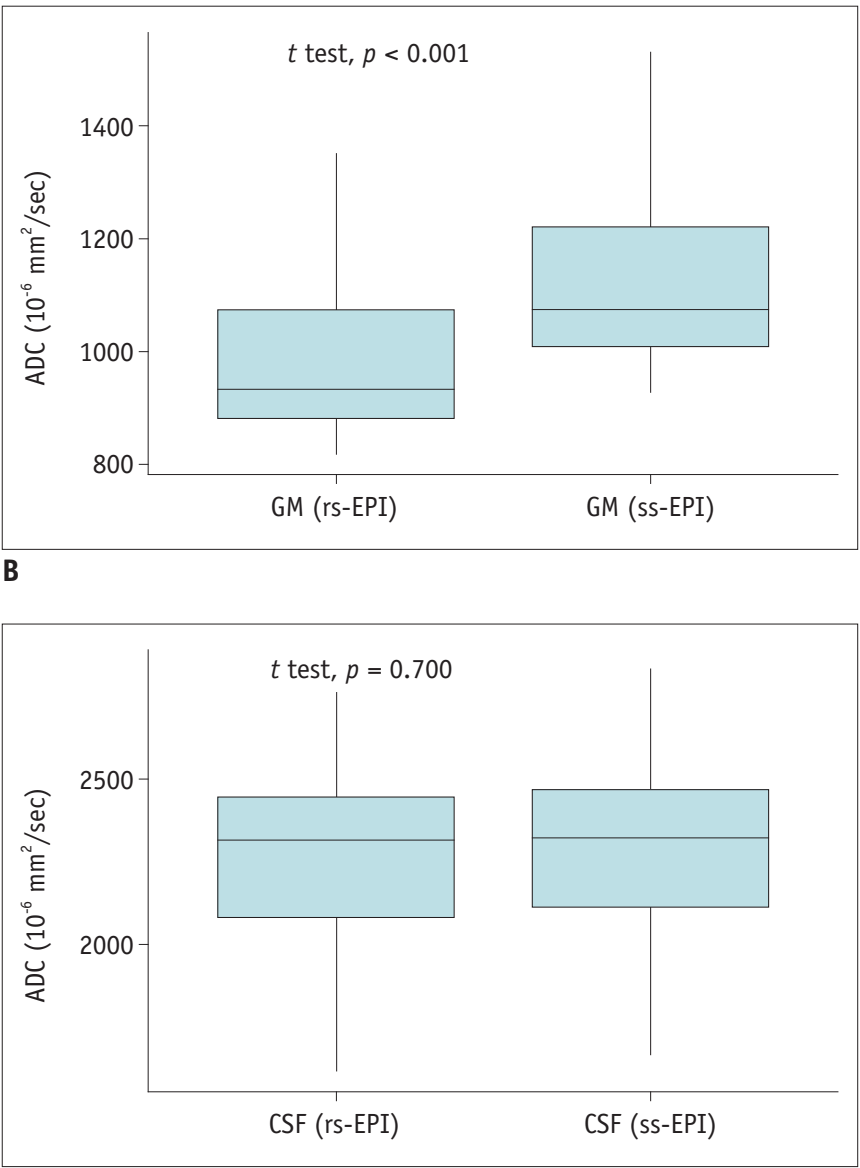

D

Fig. 2. Box plots for ADCs of rs-EPI and ss-EPI in whole brain (A), GM (B), WM (C), and CSF (D). Corresponding $p$ values between two ADCs are shown in upper left corners.

space (24). This multi-compartmental characteristic of the brain WM could also increase the sensitivity of water diffusivity to variable acquisition methods, thus producing a large variation between the WM ADC values from the two acquisition modes.

Acknowledging the differences in ADCs between ss-EPI and rs-EPI can also be important in clinical practice. In a study by Purushotham et al. (1), delineation of the ischemic core on an $A D C$ map was identified automatically with a threshold of $\leq 620 \times 10^{-6} \mathrm{~mm}^{2} / \mathrm{s}$, which yielded reasonable sensitivity and specificity. In this regard, overestimation or underestimation of infarct core lesions in patients with stroke because of the difference in ADC thresholds is possible; such inaccurate estimation may potentially affect therapeutic decisions. In oncologic treatment response evaluation, an increase in $A D C$ is considered to reflect a good response (5). Although the results of our study did not show a wide range of $A D C$ values since only healthy controls were included, the Bland-Altman plots of the GM and WM ADCs illustrated noticeable (more than 10\%) intra- individual differences. For instance, 10\% intra-individual differences of ADCs could hinder reliable estimation of infarct core sizes and evaluation of oncologic treatment responses. Hence, differences in ADCs between two different acquisition methods could pose difficulty in defining the absolute thresholds of ADCs in various diseases wherein the application of ADCs is most useful.

There are several limitations to this study. In addition to the inherent selection bias associated with a retrospective study design, only the quantitative relationship between the two ADCs from healthy subjects was evaluated. All images were acquired two dimensionally, and the generation of masks and segmentation of brain tissues could be imperfect. In addition, the examined images had a slice thickness of 5 $\mathrm{mm}$, which could have lowered the segmentation qualities owing to partial volume artifacts. However, we assumed those limitations would have equal effects on ADCs of both ss-EPI and rs-EPI sequences. Furthermore, the difference in TR and TE between the two acquisition modes could bias the results. Finally, only healthy subjects were included in 

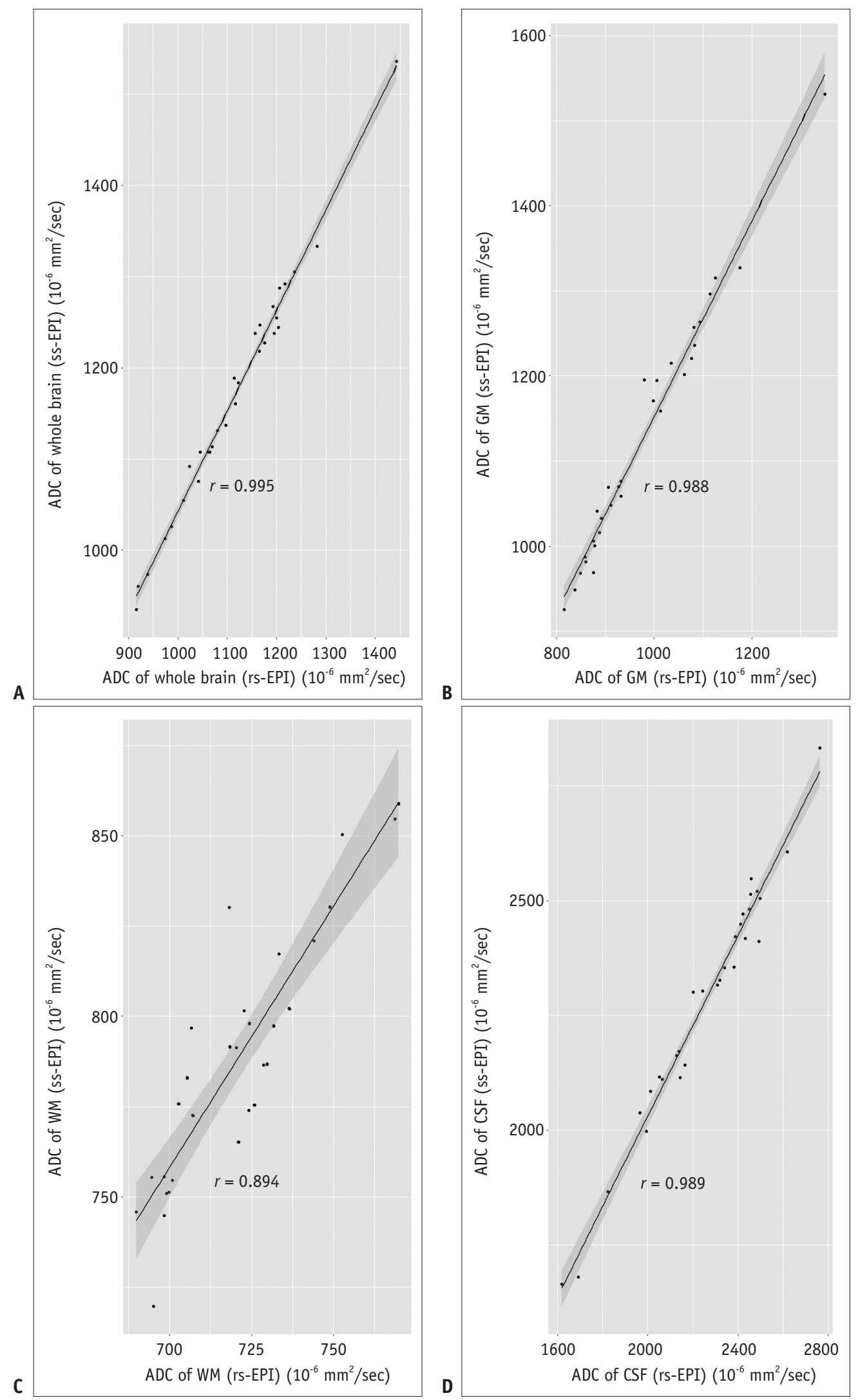

Fig. 3. Scatter plots with estimated Pearson's correlation lines and 95\% confidence intervals of whole brain (A), GM (B), WM (C), and CSF (D). 

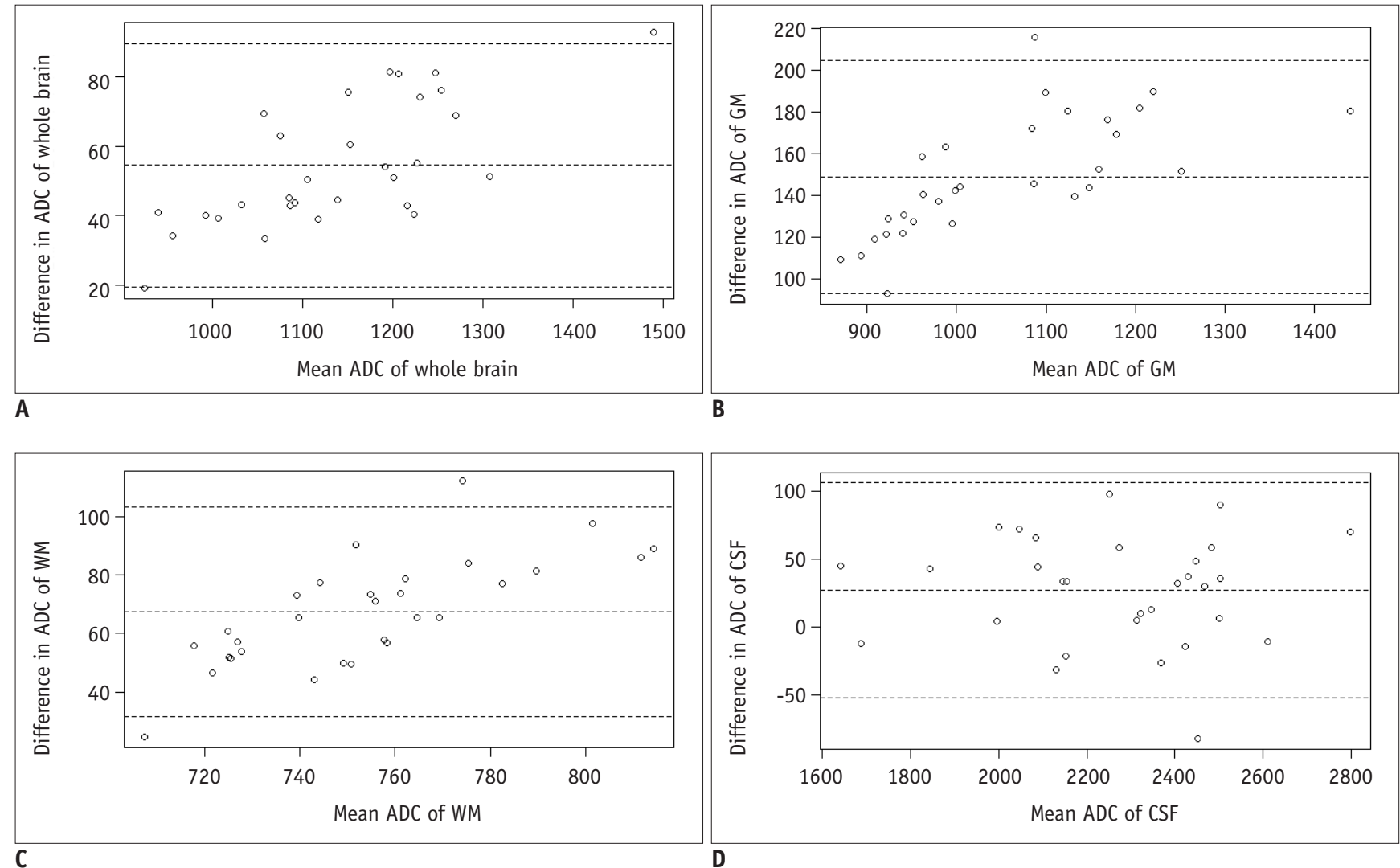

Fig. 4. Bland-Altman plots show variability in ADCs between rs-EPI and ss-EPI of whole brain (A), GM (B), WM (C), and CSF (D). Mean ADC differences and limits of agreements (1.96 times standard deviation) are displayed; all units are in $10^{-6} \mathrm{~mm}^{2} / \mathrm{s}$.

this study. Further studies enrolling patients with various diseases are strongly warranted to confirm the results of our study in pathologic conditions.

In conclusion, while ADCs of rs-EPI and ss-EPI showed high correlation and agreement in the whole brain and CSF, $A D C s$ of the WM and GM showed significant differences and large variability, reflecting brain parenchymal inhomogeneity due to different regional microenvironments. ADCs of different acquisition methods need to be interpreted carefully, especially in intra-individual comparisons. Future studies should aim to elucidate such differences.

\section{Conflicts of Interest}

The authors have no potential conflicts of interest to disclose.

\section{ORCID iDs}

Hyun Seok Choi

https://orcid.org/0000-0003-4999-8513

Yangsean Choi

https://orcid.org/0000-0003-1674-7101

\section{REFERENCES}

1. Purushotham A, Campbell BC, Straka M, Mlynash M, Olivot JM, Bammer R, et al. Apparent diffusion coefficient threshold for delineation of ischemic core. Int J Stroke 2015;10:348-353

2. Bonati LH, Lyrer PA, Wetzel SG, Steck AJ, Engelter ST. Diffusion weighted imaging, apparent diffusion coefficient maps and stroke etiology. J Neurol 2005;252:1387-1393

3. Kidwell CS, Jahan R, Gornbein J, Alger JR, Nenov V, Ajani Z, et al.; MR RESCUE Investigators. A trial of imaging selection and endovascular treatment for ischemic stroke. $N$ Engl J Med 2013;368:914-923

4. Brandão LA, Shiroishi MS, Law M. Brain tumors: a multimodality approach with diffusion-weighted imaging, diffusion tensor imaging, magnetic resonance spectroscopy, dynamic susceptibility contrast and dynamic contrastenhanced magnetic resonance imaging. Magn Reson Imaging Clin N Am 2013;21:199-239

5. Hamstra DA, Rehemtulla A, Ross BD. Diffusion magnetic resonance imaging: a biomarker for treatment response in oncology. J Clin Oncol 2007;25:4104-4109

6. Moffat BA, Chenevert TL, Lawrence TS, Meyer CR, Johnson $T D$, Dong $Q$, et al. Functional diffusion map: a noninvasive MRI biomarker for early stratification of clinical brain tumor response. Proc Natl Acad Sci U S A 2005;102:5524-5529 
7. Hayashida Y, Yakushiji T, Awai K, Katahira K, Nakayama Y, Shimomura 0, et al. Monitoring therapeutic responses of primary bone tumors by diffusion-weighted image: initial results. Eur Radiol 2006;16:2637-2643

8. Atay M, Aralasmak A, Sharifov R, Kilicarslan R, Asil T, Alkan A. Transient cytotoxic edema caused by hypoglycemia: follow-up diffusion-weighted imaging features. Emerg Radiol 2012;19:473-475

9. Kishimoto K, Tajima S, Maeda I, Takagi M, Ueno T, Suzuki N, et al. Endometrial cancer: correlation of apparent diffusion coefficient (ADC) with tumor cellularity and tumor grade. Acta Radiol 2016;57:1021-1028

10. Abdel Razek A, Mazroa J, Baz H. Assessment of white matter integrity of autistic preschool children with diffusion weighted MR imaging. Brain Dev 2014;36:28-34

11. Porter DA, Heidemann RM. High resolution diffusion-weighted imaging using readout-segmented echo-planar imaging, parallel imaging and a two-dimensional navigator-based reacquisition. Magn Reson Med 2009;62:468-475

12. Holdsworth SJ, Skare S, Newbould RD, Guzmann R, Blevins $\mathrm{NH}$, Bammer R. Readout-segmented EPI for rapid high resolution diffusion imaging at 3 T. Eur J Radiol 2008;65:3646

13. Tokoro H, Fujinaga Y, Ohya A, Ueda K, Shiobara A, Kitou Y, et al. Usefulness of free-breathing readout-segmented echoplanar imaging (RESOLVE) for detection of malignant liver tumors: comparison with single-shot echo-planar imaging (SS-EPI). Eur J Radiol 2014;83:1728-1733

14. Zhao M, Liu Z, Sha Y, Wang S, Ye X, Pan Y, et al. Readoutsegmented echo-planar imaging in the evaluation of sinonasal lesions: a comprehensive comparison of image quality in single-shot echo-planar imaging. Magn Reson Imaging 2016;34:166-172

15. Bogner W, Pinker-Domenig K, Bickel H, Chmelik M, Weber M, Helbich $\mathrm{TH}$, et al. Readout-segmented echo-planar imaging improves the diagnostic performance of diffusion-weighted MR breast examinations at 3.0 T. Radiology 2012;263:64-76

16. Li L, Wang L, Deng M, Liu H, Cai J, Sah VK, et al. Feasibility study of 3-T DWI of the prostate: readout-segmented versus single-shot echo-planar imaging. AJR Am J Roentgenol 2015;205:70-76

17. Holdsworth SJ, Yeom K, Skare S, Gentles AJ, Barnes PD, Bammer R. Clinical application of readout-segmented- echoplanar imaging for diffusion-weighted imaging in pediatric brain. AJNR Am J Neuroradiol 2011;32:1274-1279

18. Iima M, Yamamoto A, Brion V, Okada T, Kanagaki M, Togashi K, et al. Reduced-distortion diffusion MRI of the craniovertebral junction. AJNR Am J Neuroradiol 2012;33:1321-1325

19. Friedli I, Crowe LA, Viallon M, Porter DA, Martin PY, de Seigneux $S$, et al. Improvement of renal diffusion-weighted magnetic resonance imaging with readout-segmented echoplanar imaging at 3T. Magn Reson Imaging 2015;33:701-708

20. Le Bihan D, Johansen-Berg H. Diffusion MRI at 25: exploring brain tissue structure and function. Neuroimage 2012;61:324341

21. Kunz N, Zhang H, Vasung L, O'Brien KR, Assaf Y, Lazeyras F, et al. Assessing white matter microstructure of the newborn with multi-shell diffusion MRI and biophysical compartment models. Neuroimage 2014;96:288-299

22. Assaf Y, Freidlin RZ, Rohde GK, Basser PJ. New modeling and experimental framework to characterize hindered and restricted water diffusion in brain white matter. Magn Reson Med 2004;52:965-978

23. Clark CA, Le Bihan D. Water diffusion compartmentation and anisotropy at high $\mathrm{b}$ values in the human brain. Magn Reson Med 2000;44:852-859

24. Niendorf T, Dijkhuizen RM, Norris DG, van Lookeren Campagne M, Nicolay K. Biexponential diffusion attenuation in various states of brain tissue: implications for diffusion-weighted imaging. Magn Reson Med 1996;36:847-857 